\title{
Sistema Integrado de Custo Municipal: uma Ferramenta de Apoio à Tomada de Decisão pelo Gestor Público.
}

\author{
Alternative Title: Integrated System of Municipal Costs: a Tool to Support \\ Decision Making for Public Manager
}

\author{
Anderson P. Avila-Santos \\ Universidade Estadual de Londrina \\ Departamento de Computação \\ anderson.avilasantos@gmail.com \\ Evandro Baccarin \\ Universidade Estadual de Londrina \\ Departamento de Computação \\ bacarin@uel.br
}

\author{
Daniel S. Kaster \\ Universidade Estadual de Londrina \\ Departamento de Computação \\ dskaster@uel.br \\ Letícia F. Negreiros \\ Universidade Estadual de Londrina \\ Departamento de Administração \\ leticia_negreiros@hotmail.com
}

\section{Saulo F. Amâncio Vieira \\ Universidade Estadual de Londrina \\ Departamento de Administração \\ saulofav@gmail.com}

\begin{abstract}
RESUMO
Este artigo tem como objetivo demonstrar a eficácia de uma ferramenta de apoio ao controle social da gestão pública, denominada SICM-Educação (Sistema Integrado de Custos Municipais - Educação), que foi desenvolvida pela Universidade Estadual de Londrina em um trabalho conjunto do Departamento de Computação e do Departamento de Administração. O propósito dessa ferramenta é possibilitar a visualização e a comparação dos custos das escolas, por exemplo, dentro de um município. O artigo apresenta um estudo de caso utilizando a ferramenta proposta sobre dados obtidos a partir de um dos levantamentos realizados, referentes ao município de Assaí/PR. Também é mostrado como os dados podem ser visualizados através da ferramenta e como eles podem ser utilizados em tomadas de decisão por parte do poder público, o que se torna um poderoso meio de impor a transparência e a fiscalização sobre a administração pública.
\end{abstract}

\section{Palavras-Chave}

Controle social, tomada de decisão, governo eletrônico

*Este trabalho teve apoio financeiro da SETI - Secretaria da Ciência, Tecnologia e Ensino Superior do Paraná.

Permission to make digital or hard copies of all or part of this work for personal or classroom use is granted without fee provided that copies are not made or distributed for profit or commercial advantage and that copies bear this notice and the full citation on the first page. To copy otherwise, to republish, to post on servers or to redistribute to lists, requires prior specific permission and/or a fee.

SBSI 2015, May 26th-29th, 2015, Goiânia, Goiás, Brazil

Copyright SBC 2015.

\begin{abstract}
This paper aims at demonstrating the effectiveness of a support tool for the social control of public management, the so-called SICM-Educação (Integrated System of Municipal Costs - Education), which has been developed at the University of Londrina in a joint effort of the Department of Computing and the Department of Administration. The purpose of such a tool is to allow visualizing and comparing costs of schools, e.g. within a city. The paper shows a case study using the proposed tool over data obtained from one of the conducted surveys, regarding the city of Assaí/PR. It is also shown how the tool can be used to visualize the collected data and how it can be used to aid decision-making in public power, which enforces transparency and citizens control over the public administration.
\end{abstract}

\section{Categories and Subject Descriptors}

J.1 [Administrative data processing]: GovernmentEducation, Financial

\section{General Terms}

Education, transparency, administration

\section{Keywords}

Social control, decision-making, e-government

\section{INTRODUÇÃO}

A partir da década de 1990, o Estado brasileiro, acompanhando o movimento global de modernização do Estado, passa a ser pressionado a inserir práticas de cunho mais gerencial. Neste mesmo contexto, desde então passa-se a exigir cada vez mais nas decisões públicas a transparência, a eficiência e a possibilidade de controle social. No entanto, faltam 
ferramentas que deem suporte a essas novas demandas. Ferramentas como softwares de gestão seriam de grande valia para subsidiar as ações dos gestores e para gerar à população feedbacks em uma linguagem comum à dos cidadãos, ultrapassando assim a barreira que os termos técnicos de cada área impõe.

Um aspecto que há anos vem sendo discutido na gestão pública é o controle de custos. Dada a sua importância, a inserção da contabilidade de custos já estava prevista legalmente desde 1964, porém iniciou-se com o uso de tecnologia da informação apenas em 2010, com a homologação da primeira versão do Sistema de Informação de Custos do Governo Federal [19]. No entanto, a discussão de custos no âmbito municipal ainda é ausente.

Sabe-se da necessidade de se apurar os custos mas pouco ainda se sabe como fazer essa apuração e de que forma utilizar esses resultados para a melhoria da execução dos serviços. O problema é ainda mais preocupante na esfera municipal, uma vez que esta tem ganho maior responsabilidade na execução dos serviços e, por consequência, tem recebido maior repasse de verbas da União. Na década de 1990 foi realizado o processo de descentralização administrativa por parte da União aos Estado e Municípios porém, sem que houvesse o adequado treinamento e municiamentos destes entes federativos com ferramentas de gestão que os auxiliassem nos processos de controle e gerenciamento administrativo.

Outro ponto importante a se destacar relaciona-se a utilização de mecanismos informacionais que possam contribuir para uma maior participação social no acompanhamento da prestação de serviços públicos. Mesmo previsto em lei, Lei de Acesso a Informação (LAI), LEI N ${ }^{\mathrm{O}} 12.527$, verifica-se uma baixa adesão por parte dos municípios na utilização de ferramentas que melhorem a transparência na gestão dos recursos públicos e por conseguinte uma maior participação social.

Neste contexto, este artigo apresenta o desenvolvimento de um software de apuração de custos municipais para o Ensino Fundamental e seus benefícios como ferramenta de apoio à tomada de decisão. Esta pesquisa descreve o desenvolvimento do software, e apresenta os resultados preliminares de um município que fez uso do sistema.

Esta ferramenta está no momento sendo utilizada por vários municípios que estão em fase de inserção dos dados. Ao finalizarem esta etapa já poderão usufruir dos relatórios e análises que estarão disponíveis aos municípios e aos cidadãos. Estas informações podem ser utilizadas tanto para a tomada de decisão por parte dos orgãos públicos, quanto a nível de fiscalização por parte da população que poderá avaliar como está sendo gasto os recursos com educação do seu município e do estado.

O artigo está dividido em cinco partes. Há Gestão de custos na administração pública no brasil, apresenta a atual situação do Brasil com relação a sistemas desse tipo. Na sequencia existe a parte de Sistemas de apoio à gestão de custos educacionais, qual aborda os sistemas de apoio à tomada de decisão como auxílio à gestão de custos na educação. Em seguida têm-se a apresentação do software, onde é apresentada a motivação para a criação do software e como o software foi desenvolvido. Próxima parte, os resultados preliminares, apresenta alguns resultados e contribuições à gestão que software pode fornecer. Por fim, tem-se as considerações finais do artigo.

\section{GESTÃO DE CUSTOS NA ADMINISTRA- ÇÃO PÚBLICA NO BRASIL}

A administração pública visa gerenciar os recursos públicos com o objetivo de atender às necessidades coletivas. Para tanto, a administração pública é regida por cinco princípios constitucionais que dão a base para a sua condução. Esses cinco princípios são: legalidade, impessoalidade, moralidade, publicidade e eficiência. O primeiro dos princípios, a legalidade, diz respeito à subordinação dos gestores públicos à lei, atuando somente quando esta permitir. O princípio da impessoalidade, por sua vez, busca garantir que o administrador público atue independentemente da pessoa que será atingida ou favorecida pela sua decisão. O terceiro dos princípios é a moralidade, que relaciona-se com a moralidade jurídica, a negação da corrupção e de outros atos ilegais. Já a publicidade, visa que os atos administrativos devem ser objeto de ampla divulgação. E, por fim, a eficiência compreende a otimização dos recursos públicos, buscando a melhor relação entre custo e benefício [25].

Segundo Alonso [2], com a intensificação da crise fiscal do Estado e a maior exposição da economia nacional à competição internacional, o Estado foi forçado a reorientar a política econômica e, em particular, conter suas despesas. Inicialmente, a concepção adotada passou a ser cortar gastos, mas, com a exaustão dessa política, organismos internacionais, como o Banco Mundial e o Fundo Monetário Internacional (FMI), vêm incentivando o aumento da qualidade dos gastos públicos e não simplesmente o seu corte.

A partir dos anos de 1990 tem-se buscado gradativamente inserir práticas gerenciais, advindas principalmente da iniciativa privada, se tem denominado de administração pública gerencial. Este novo modelo de gestão visa atender aos novos desafios de um mundo globalizado, buscando meios para mudar a visão atual que se tem do Estado, ente lento e ineficiente [6]. A administração pública gerencial enfatiza a produtividade, a eficiência, a descentralização administrativa, o corte dos custos públicos, a implementação de instrumentos gerenciais, o foco no cidadão, a preocupação com a qualidade do serviço público e a maior participação e avaliação dos cidadãos perante a gestão pública $[23,1]$.

A contabilidade de custos gera informações gerenciais que permitem aos diversos níveis hierárquicos da administração sejam capazes de planejar, controlar e decidir com maior eficiência e eficácia [15]. No que tange a adminstração pública no Brasil, a Lei de Responsabilidade Fiscal (LRF) instituiu normas de finanças públicas e estabelece no parágrafo $3^{\circ}$ do Art. 50 que "A Administração Pública manterá sistema de custos que permita a avaliação e o acompanhamento da gestão orçamentária, financeira e patrimonial". É consenso a necessidade de sistemas de informação para implantar essas ações. Entretanto, devido à ausência de rotinas de responsabilização e incentivos na Administração Pública, muitos gestores sentem-se desconfortáveis pela possibilidade de serem avaliados por seu desempenho ou decisões quanto à alocação de recursos. Além disso, as informações disponibilizadas são de difícil entendimento pelo público e, como consequência, o contribuinte não consegue identificar ou rastrear a aplicação dos recursos arrecadados [19].

O sistema mais importante existente nesse contexto é o Sistema de Informação de Custos do Governo Federal (SIC), desenvolvido pela Secretaria do Tesouro Nacional do Ministério da Fazenda em conjunto com o Ministério do Planeja- 
mento, Orçamento e Gestão. O SIC é um data warehouse que capta informações transacionais dos chamados Sistemas Estruturadores do Governo Federal (Sigplan, Sidor, Siafi, Siape, Siasg e outros), com intuito de apurar os custos dos programas e unidades da Administração Pública Federal e permitir o acompanhamento e avaliação da gestão orçamentária, financeira e patrimonial do Governo Federal, como rege a LRF [14]. Embora o SIC seja uma grande evolução, ainda há uma enorme carência de sistemas de informação de custos, particularmente na esfera municipal e, no contexto desse trabalho, para a área de educação, como apresenta a próxima seção.

\section{SISTEMAS DE APOIO À GESTÃO DE CUSTOS EDUCACIONAIS}

Para alcançar a gestão pública gerencial é necessário implementar e utilizar ferramentas que possibilitem informações para subsidiar as decisões dos gestores. Nesse sentido ressalta-se a relevância que os sistemas de apoio a decisão têm neste contexto da administração pública. Sistema de apoio a decisão (Decision Support Systems - DSS) têm por objetivo disponibilizar informações que possam auxiliar numa melhor tomada de decisão, é essencialmente baseada na implantação de sistemas informatizados [4].

Existem vários trabalhos na literatura que abordam a eficiência de DSS em gestão pública, nas mais diversas áreas. Trabalhos que focam na gestão de custos na administração pública em geral, como o de Mello [18] que propõe um modelo de método de custeio e a utilização de um sistema para gerenciamento do custo, demonstrando que a utilização desse tipo de sistema desencadeia uma evolução da estrutura orçamentária, visto que este ajuda numa melhor tomada de decisão na maneira como são gastos os recursos disponíveis, mas são poucas as propostas de sistemas que abordem os níveis munícipais.

Temos também alguns trabalhos que abordam áreas mais específicas como a área da saúde, o trabalho apresentado por Martinez e Alves Filho [17] apresenta um caso real de implantação de um sistema de custos em uma Secretaria Municipal de Saúde, este trabalho demonstrou algumas motivações para implantação de sistemas desse tipo, entre elas estão o dever de prestar contas de maneira transparente para a sociedade e a profissionalização da gestão pública, utilizando esse tipo de sistema para a melhoria do processo e pllanejamento, redução da capacidade ociosa e controle dos gastos com as atividades realizadas, na área da saúde ainda existem mais alguns trabalhos que estudam a gestão dos custos $[10,13]$.

Podemos encontrar também alguns poucos trabalhos que abordam a questão dos custos na educação, temos por exemplo, trabalhos que apresentam a questão do custo em instituição estadual de ensino superior no estado do Paraná, Moraes e Calijuri [20] ralataram a implementação de um sistema de custos nessas instituições realizando a vinculação dos valores investidos com os resultados que se esperados, buscando verificar a eficiência na aplicação dos recursos.

Um trabalho que se aproxima um pouco da nossa proposta, foi apresentado por Alves e Martinez [3] eles avaliaram que sistemas desse tipo tornam possível a profissionalização, eficiência e efetividade na gestão dos custos, algo que está se tornando cada vez mais recorrente no meio público. Este trabalho buscou destacar é a importância ad- ministrativa desse instrumento, para estimular e ampliar as possibilidades do gestor público na condução econômica e financeira do patrimônio público, principalmente através da otimização da função controle. Com isto foi possível realizar a mensuração e um monitoramento sistemático de seu desempenho econômico e financeiro, estas entidades públicas podem dar um grande salto de qualidade. Com a utilização de um sistema de custos, a avaliação do desempenho poderia ser melhorado através do monitoramento de informações e indicadores.

Entretanto, quando atinge-se a capilaridade municipal, o uso de sistemas computacionais de apoio cai drasticamente, em especial em municípios de menor porte. Por exemplo, o trabalho de Almeida et al. [11] traçou um perfil da utilização de informações de custos na gestão de saúde pública em secretarias municipais de saúde do estado de Santa Catarina. Das 18 secretarias municipais de saúde pesquisadas, apenas 2 possuíam esse processo um pouco mais avançado. Nas demais, foi verificado que existem apenas algumas tentativas de se obter informações mais detalhadas sobre custos. Vale ressaltar que Santa Catarina apresentou o terceiro maior índice de desenvolvimento humano municipal (IDHM) em 2013 dentre todos os estados brasileiros, segundo o Atlas do Desenvolvimento Humano do Brasil ${ }^{1}$.

Ora, esses tipos de sistemas são utilizados com grande frequência no mundo corporativo, os dados gerados em processos organizacionais podem ser observados de duas maneiras, uma delas é a necessidade de técnicas eficientes para a interpretação dos dados e a outra a grande demanda por sistemas que garantam a transparencia dos dados [22]. Assim chegamos ao conceito de transparência de dados. A utilização de dados abertos para promover a transparência, vem com a possibilidade de tornar esses acessíveis a quem tiver interesse, eliminando as restrições de tecnologia, legislação e acessibilidade, garantindo assim o acesso desses dados pelo cidadão [24].

\section{APRESENTAÇÃO DO SOFTWARE}

O software foi desenvolvido após os trabalhos de $[7,5,21$, $8,16]$ demonstrarem a importância do controle de custos e que uma gestão de custos eficiente melhora o desempenho dos alunos da Educação fundamental. Em comum, esses trabalhos identificaram a necessidade de obtenção de mais dados para a realização de análises mais precisas neste contexto. Como consequência, constituiu-se uma equipe multidisciplinar envolvendo docentes e discentes de cursos de graduação e pós-graduação do departamentos de Computação e Administração da Universidade Estadual de Londrina, desenvolvido em convênio com a Secretaria da Ciência, Tecnologia e Ensino Superior (SETI) do Paraná. Esta equipe iniciou o projeto e implementação do Sistema Integrado de Custos Municipais (SICM).

O primeiro módulo desenvolvido, denominado SICM Educação, é voltado à gestão de custos referentes aos anos iniciais do Ensino Fundamental. Este sistema colige os fatores que mais contribuem para o custo total do Ensino Fundamental, a saber, o custo de pessoal e merenda. É notável a ausência do fator transporte no cômputo do custo total. Esta ausência se deve ao fato de que os veículos usados no transporte escolar também são usados para outras finalidades, não se podendo aferir a parcela correspondente a cada

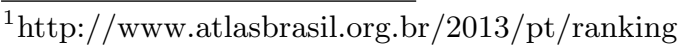


finalidade. Este módulo tem por objetivos:

- Levantar dados dos custos das unidades escolares dos municípios do Paraná;

- Possibilitar cálculos e comparações dos custos das escolas;

- Identificar as correlações entre custos específicos (e seus respectivos elementos) de um ano exercício com o desempenho escolar aferido posteriormente por exames de avaliação como o IDEB.

O IDEB foi criado pelo INEP (Instituto Nacional de Estudos e de Pesquisas Educacionais Anísio Teixeira) em 2007, ele é calculado levando em conta a taxa de rendimento escolar - aprovação e evasão - considerando também o desempenho dos alunos no SAEB (Sistema Nacional de Avaliação da Educação Básica) e na Prova Brasil. Por ser algo realizado no âmbito nacional, podemos utiliza-la para avaliação do desempenho das escolas.

A gestão de custos e transparência à população é comprometida pelo fato das informações estarem espalhadas em diversos orgãos do município (Figura 1). Vale observar que não existe, a priori, nenhum esquema de integração, validação, consistência, controle, etc destas diversas fontes. Por isso, o SICM-Educação as centraliza, provendo aos gestores e à população fácil acesso a estes dados, visando ações gerenciais ou permitindo o controle dos gastos públicos pelo cidadão.

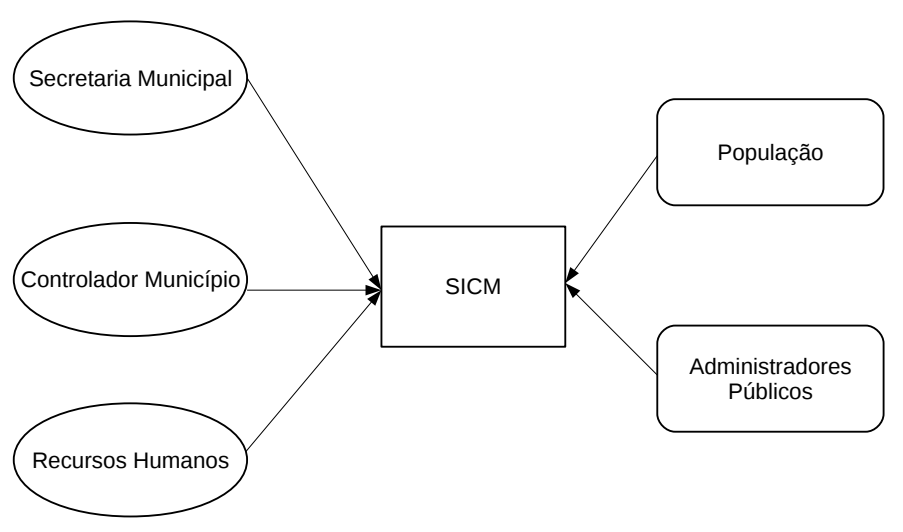

Figura 1: Distribuição dos dados nos municípios

Os dados são obtidos manualmente das diversas fontes e, também manualmente, são inseridos no SICM-Educação. Para as maiores cidades do estado foram desenvolvidos scripts individuais para a importação automática. Após os dados de um ano exercício terem sido informados, o preenchimento é finalizado, podendo, assim, serem produzidos diversos tipos de relatórios. Tais relatórios podem, por exemplo, mostrar grandes discrepâncias nos custos de diferentes escolas da mesma cidade. Estas discrepâncias podem ocorrer por diversos motivos e podem indicar que ações gerenciais são necessárias.

O sistema possui quatro tipos de usuários com diferentes níveis de acesso, a saber:

- Visitante: permite ao cidadão produzir relatórios sobre os custos de uma escola ou de um muncípio específicos.
- Controlador do município: é um funcionário da secretaria municipal de educação, responsável pelo preenchimento dos dados. Tem acesso a outros relatórios gerenciais.

- Sub-Controlador do município: é um funcionário delegado pelo controlador para auxiliar no preenchimento dos dados.

- Administrador do sistema: tem os acesso aos cadastros, registros e log do sistema. Tém acesso a relatórios específicos a sua função.

Para o desenvolvimento do sistema foram utilizadas várias tecnologias de código aberto, tais como: Java EE, Apache Velocity, PostgreSQL, Spring Framework e Jasper Reports Library.

\section{RESULTADOS PRELIMINARES}

Após o lançamento do SICM-Educação, entrou-se em contato com os controladores municipais dos 399 municípios do Paraná, apresentando o projeto e informando o seus respectivos logins para utilização da ferramenta. A escolha pelo controlador deve-se pelo mesmo estar habituado a prestar contas ao Tribunal de Contas do Estado e, incumbido de tal atividade, é rotineiro que o controlador solicite dados das demais secretarias/departamentos da prefeitura. Essa foi a maneira encontrada para centralizar a responsabilidade do preenchimento do sistema em um único servidor. Como o preenchimento dos dados é uma tarefa facultativa, pois é uma atividade de um projeto piloto e não uma determinação do Tribunal de Contas do Estado, não houve adesão de todos os municípios. Entretanto, vários aderiram ao projeto, incluindo todos os municípios de maior porte no estado, e forneceram seus dados por meio do sistema.

Os dados solicitados aos municípios foram referentes ao ano de 2013, ano em que foi publicado o resultado mais recente do IDEB, para que fosse possível fazer uma correlação entre o custo e o desempenho de cada unidade escolar considerada. No momento da escrita deste artigo, o preenchimento dos dados não estava concluído. Desta forma, a seguir serão apresentados os resultados preliminares de Assaí, município que já finalizou o cadastro no sistema, a fim de demonstrar algumas maneiras de utilizar as informações geradas pelo sistema. Em um projeto anterior, foi feita uma coleta de dados semelhante referente ao ano de 2011, mas utilizando-se planilhas de cálculos. Como essa forma de coleta tem uma escalabilidade reduzida, apenas os municípios da Região Metropolitana de Londrina foram considerados nessa oportunidade, incluindo Assaí. O SICM-Educação foi projetado para permitir o armazenamento de dados de anos diferentes, para possibilitar análises da evolução histórica das variáveis coletadas. Portanto, os dados de 2011 coletados no projeto anterior também foram inseridos no SICM-Educação.

O município de Assaí está localizado no Norte Pioneiro Paranaense e faz parte da Região Metropolitana de Londrina. A cidade possuía em 2014, de acordo com a estimativa do Instituto Brasileiro de Geografia e Estatística (IBGE), 16.322 habitantes [12]. Assaí faz parte dos 23,1\% municípios mais desenvolvidos na área de Educação do Paraná, segundo Índice Firjan de Desenvolvimento Municipal (IFDM) [9]. Conforme a referida fonte, em termos de educa- 
ção, Assaí ocupa a $1131^{\circ}$ posição nacional e a $62^{\circ}$ posição em âmbito estadual referente ao desenvolvimento educacional.

Após o preenchimento dos dados, o SICM-Educação fornece alguns relatórios aos usuários, tanto com apresentação na tela do sistema como uma versão em PDF adaptada para impressão. A Figura 2 mostra a versão em PDF do relatório de conferência do município de Assaí referente ao ano de 2013, disponibilizado ao controlador. Considerando apenas a educação fundamental (anos iniciais), o relatório mostra, entre outras informações, que em 2013 o valor total da subfunção Ensino Fundamental de Assaí foi de quase 6 milhões e 400 mil reais e que a cidade possuía seis unidades escolares para atender a 997 alunos do mesmo nível de ensino.

\section{SICM}

Relatório de conferência

\begin{tabular}{|c|c|c|c|c|c|c|c|}
\hline 04/03/2015 & & & & & & & ASSA \\
\hline Número de alunos & & & & & & Custos (R & \\
\hline Educação infantil & 753 & & & 0,30 & Valor $\mathrm{m}$ & tio por refeici & \\
\hline Fundamental ( $1^{19}$ a $5^{\mathrm{a}}$ série) & 997 & & & & & & \\
\hline Fundamental ( $6^{\mathrm{a}} \mathrm{a} 9^{\mathrm{a}}$ série) & 0 & & 150.9 & & Adm. dz & Secret. da Ec & :açăao \\
\hline EJA & 54 & & 6.389 .5 & & Total da & ubfun. do en & fund. \\
\hline Outros alunos & 25 & & & & & & \\
\hline Total de alunos & 1829 & & 8.160 .3 & & Total da & unção da ed & ação \\
\hline Escola & & № alunos & № profs. & & funcs. & № refs. & IDEB \\
\hline AUGUSTA G ROCHA E M PROF & EIEF & 17 & 7 & & 4 & 3400 & - \\
\hline FRANCA WOLKERS E R M PE EI & & 76 & 17 & & 6 & 45600 & - \\
\hline IZABEL E M PRINC EI EF & & 198 & 21 & & 4 & 39600 & 6 \\
\hline MARIA JOSE S SANTOS E M PFA & AEI EF & 304 & 23 & & 6 & 60800 & 5,5 \\
\hline MARIA M TSUBOI E M PROFA EI & & 274 & 26 & & 7 & 54800 & 5,5 \\
\hline ROTARY CLUB E M ED INF ENS & FUND & 128 & 29 & & 4 & 76800 & 5,1 \\
\hline Secretaria Municipal de Educaçăo & & 0 & 0 & & 26 & 0 & - \\
\hline & Totais & 997 & 123 & & 57 & 281000 & 5,52 \\
\hline
\end{tabular}

Figura 2: Relatório de conferência do município de Assaí no exercício 2013.

No que tange aos custos calculados pelo sistema, enquanto a maioria dos trabalhos existentes que tratam de custos na educação partem da média de valores agregados, a metodologia adotada no sistema utiliza o custo direto, entendendo a escola como o nível micro de análise. Essa decisão é fundamentada em pesquisas que se utilizaram de custo direto, como $[7,5,21,8,16]$, e mostraram que há elevado coeficiente de variação do custo/aluno dentro de um mesmo município, evidenciando o viés de pesquisas que utilizam custos médios.

Este custo é desassociado no sistema de acordo com a finalidade de aplicação dos recursos. Os custos são classificados em custo pedagógico, administrativo e social. Entende-se por custo pedagógico os custos mensais por aluno com remuneração dos professores. Os custos sociais referem-se ao valor gasto mensalmente por aluno com merenda (remuneração dos funcionários que atuam na cozinha e custos dos gêneros alimentícios). Por fim, os custos administrativos englobam a remuneração mensal, dividido pelo número de alunos, dos outros funcionários que atuam para manter a escola, como os técnico-administrativos, os da limpeza, entre outros. O custo total é a soma destes três custos.

A Figura 3 apresenta o relatório de custos mensais de Assaí, que permite analisar os custos por aluno específicos de cada unidade escolar. Analisando o relatório, conclui-se que o caso de Assaí corrobora com os estudos que defendem o uso de custo direto e não valores agregados ao apontar como os custos com as unidades escolares do município variam significativamente. Enquanto a Escola Maria José Silva Santos tem um custo por aluno de $\mathrm{R} \$ 126,73$ mensais, a Escola Prof. Augusta Gino Rocha desembolsa R\$ 629,92 a cada aluno mensalmente, o que representa um custo $496 \%$ superior que a escola anterior.

A separação do custo segundo sua finalidade também permite comparações entre os recursos mais representativos dentro de cada unidade. É de se esperar que nas escolas o custo mais elevado seja mesmo com remuneração de professores (custo pedagógico). Todavia, com os dados gerados pelo sistema pode-se notar que algumas escolas estão mais "inchadas" em termos administrativos do que outras. Por exemplo, do total gasto por aluno na Escola Rotary Club o custo administrativo corresponde a $8 \%$ enquanto na Escola Prof. Augusta Gino Rocha este custo chega a 23\% do total. Somente o custo administrativo desta última escola $(\mathrm{R} \$ 143,89)$ ultrapassa o valor total de um aluno que estuda na Escola Maria José Silva Santos (R \$ 126,73).

No entanto, sempre há de se fazer ressalvas sobre análises desses dados financeiros como o aqui apresentados. Por isso, é importante incluir outras variáveis, além das de custos, na tentativa de entender melhor a realidade de cada escola. Exemplos de variáveis que podem auxiliar na explicação da discrepância de custo e que encontram-se presentes no SICM-Educação são as seguintes: o número de alunos atendidos pela escola, o número de merendas servidas, a questão do atendimento da escola - caso seja de tempo integral - ou ainda, se está localizada no meio rural. Ao considerar esses outros dados percebe-se em Assaí, conforme preenchimento do sistema, que a escola com maior custo (Escola Prof. Augusta Gino Rocha) é também a que possui menor número de alunos (vide Figura 2). Da mesma forma, a escola com menor custo total por aluno (Escola Maria José Silva Santos) é a que atende a um maior número de estudantes, o que provoca economia de escala.

Outra possibilidade fornecida pelo SICM é a comparação entre as variáveis de custo por aluno mensal (custo total, pedagógico, social, administrativo) com o desempenho dos alunos, representado no sistema pelo Índice de Desenvolvimento da Educação Básica (IDEB). A Figura 4 mostra um gráfico comparativo entre o custo total por aluno mensal com o IDEB das escolas de Assaí dos anos de 2011 e 2013, obtido a partir de dados armazenados no sistema. Três escolas não participaram do IDEB em 2011 e duas não participaram do IDEB em 2013, isto porque escolas com menos de 20 alunos nas séries finais não participam da avaliação. Essas escolas são as que possuem menor número de alunos e também são as de custo mais elevado. Percebe-se, também, que não houve uma relação direta entre o IDEB e o custo total por aluno. Por exemplo, a Escola Princesa Izabel teve o maior IDEB nos dois anos, mesmo sendo a terceira escola de menor custo por aluno em 2011 e a segunda em 2013. Um bom indicativo é que todas as escolas que participaram do IDEB nos dois anos tiveram aumento no índice e menor variação no custo por aluno de uma coleta para a outra $(9,5 \%,-7,5 \%$ e $3,3 \%$, respectivamente para as escolas Maria Mitiko Tsuboi, Maria José Silva Santos e Princesa Izabel).

As escolas Prof. Augusta Gino Rocha e Rotary Club apresentaram uma variação significativa de custo por aluno devido à maior redução relativa no número de alunos, comparando-se com as demais escolas. Isso pode ser visto no gráfico da Figura 5, que apresenta a evolução do custo total 


\section{Relatório de custos mensais}

\section{ATENÇĀO: SOMENTE CONSIDERAR DADOS DO ENSINO FUNDAMENTAL (ANOS INICIAIS - 1ํ. AO 5‥ ANO) DE 2013}

\begin{tabular}{l|l|l|l|l}
\multicolumn{2}{c}{ Relatório de custos mensais } & & \\
\hline Escola & Custo Pedagógico & Custo Administrativo & Custo Social & Custo Total \\
\hline AUGUSTA G ROCHA E M PI R $\$ 480,03$ & $\mathrm{R} \$ 143,89$ & $\mathrm{R} \$ 5,00$ & $\mathrm{R} \$ 628,92$ \\
\hline FRANCA WOLKERS E R M I $\mathrm{R} \$ 247,26$ & $\mathrm{R} \$ 69,69$ & $\mathrm{R} \$ 15,00$ & $\mathrm{R} \$ 331,95$ \\
\hline IZABEL E M PRINC EI EF & $\mathrm{R} \$ 126,20$ & $\mathrm{R} \$ 13,69$ & $\mathrm{R} \$ 5,00$ & $\mathrm{R} \$ 144,89$ \\
\hline MARIA JOSE S SANTOS E N R\$ $\mathrm{R} 102,53$ & $\mathrm{R} \$ 19,20$ & $\mathrm{R} \$ 5,00$ & $\mathrm{R} \$ 126,73$ \\
\hline MARIA M TSUBOI E M PRO $\mathrm{R} \$ 121,49$ & $\mathrm{R} \$ 21,79$ & $\mathrm{R} \$ 5,00$ & $\mathrm{R} \$ 148,27$ \\
\hline ROTARY CLUB E M ED INF & $\mathrm{R} \$ 243,88$ & $\mathrm{R} \$ 21,96$ & $\mathrm{R} \$ 15,00$ & $\mathrm{R} \$ 280,83$
\end{tabular}

Data atual: $04 / 03 / 2015$

\section{$\checkmark$ Gerar PDF Gerar CSV}

Figura 3: Relatório de custos mensais por aluno de Assaí no exercício 2013.

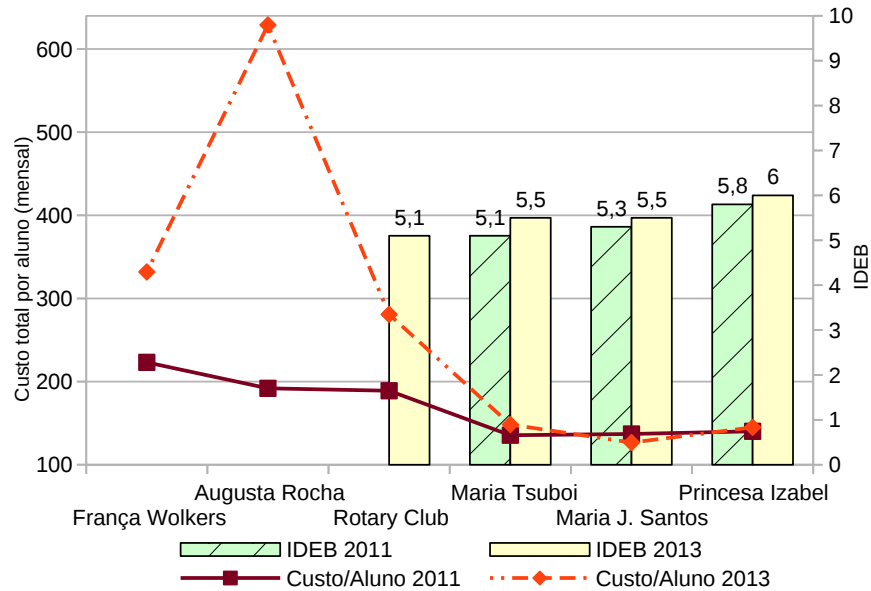

Figura 4: Relação entre custo total por aluno e IDEB de 2011 e 2013 das escolas de Assaí.

mensal e do número de alunos das escolas de 2011 a 2013. Em particular, a variação abrupta do custo por aluno da escola Prof. Augusta Gino Rocha identificada na Figura 4 (aumento de $227 \%$ ), deveu-se ao fato de a quantidade de alunos ter caído $89 \%$ enquanto seu custo total mensal diminuiu apenas $63,8 \%$. A Figura 5 também mostra uma relação direta entre o custo da escola e a sua quantidade de alunos. Observou-se uma queda no número de alunos entre os anos e, consequentemente, uma queda no custo total das escolas, com exceção da Escola Pe. França Wolkers, que teve um aumento do custo total mensal de $12 \%$ mesmo com uma queda de quase $25 \%$ no número de alunos.

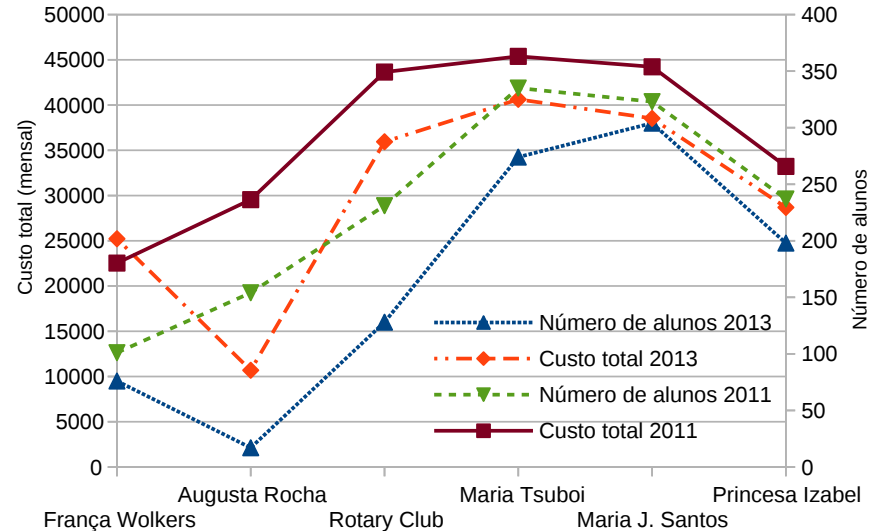

Figura 5: Relação entre custo total e número de alunos de 2011 e 2013

Buscou-se neste tópico ilustrar alguns resultados da aplicação do SICM-Educação. Entretanto, destaca-se a necessidade de estudos mais abrangentes, considerando outros municípios, para que se possa ter constatações mais fundamentadas. Também neste prisma o sistema poderá contribuir para a área acadêmica, ao criar um banco de dados inédito sobre o tema.

Além de análises internas, o SICM-Educação disponibiliza relatórios públicos, que permitem que o cidadão tenha acesso aos custos e desempenhos das escolas, em diferentes níveis de agregação (estado, município e escola). A Figura 6 apresenta o relatório da Escola Maria Mitiko Tsuboi e os indicadores gerais de Assaí. O mapa apresentado na figura 


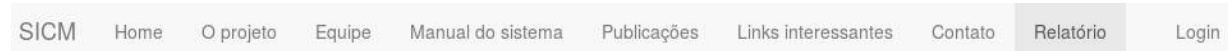

\section{Relatório geral}

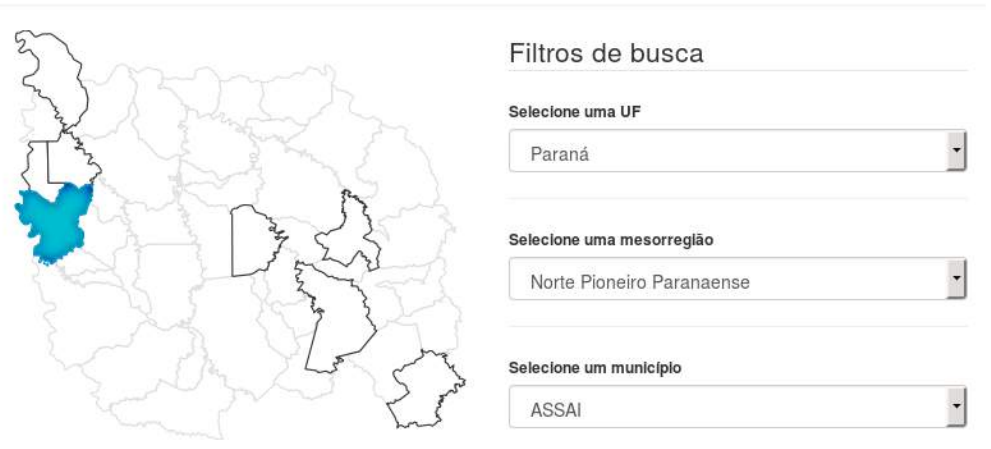

\begin{tabular}{|l|c|}
\hline Pré-Visualização do relatório geral & c Gerar PDF \\
\hline Indicadores do município & Custos \\
\hline Custo médio da escola por aluno (mensal) & $\mathrm{R} \$ 276,00$ \\
\hline Escola com maior custo por aluno (mensal) & $\mathrm{R} \$ 628,00$ \\
\hline Escola com menor custo por aluno (mensal) & $\mathrm{R} \$ 126,00$ \\
\hline IDEB Médio Munícipal & 5.53 \\
\hline Selecione uma escola & \\
\hline MARIA M TSUBOI E M PROFA EI EF & \\
\hline Indicadores da escola & IDEB da escola selecionada \\
\hline Custo total por aluno (mensal) & \multicolumn{2}{|c|}{$\mathrm{R} \$ 148,27$} \\
\hline IDEB da escola selecionada & \multicolumn{2}{|c|}{5.5} \\
\hline
\end{tabular}

Figura 6: Relatório geral de custos mensais por aluno acessível à população (município Assaí, ano 2013).

destaca os municípios da mesorregião selecionada que têm relatório disponível e destaca o município selecionado em azul. A seleção pode ser feita clicando-se direto no mapa (sequência estado, mesorregião e município) ou usando as caixas de seleção ao seu lado. O relatório público também permite a gestores verificar os resultados de escolas de outras cidades, de porte e contexto que julgar ser semelhantes ao seu como meio de benchmarking. Assim, os gestores poderiam conhecer as práticas das escolas com melhores resultados a fim de implantar em seu município, tendo cautela quanto às comparações entre escolas de cidades distintas. Nota-se que dados de cunho gerencial como estes podem auxiliar na tomada de decisões dos gestores e na transparência para a população, uma vez que não exige conhecimento técnico para interpretação dos mesmos. Nesse sentido, o SICM busca contribuir ao gerar informações que deem suporte ao gestor público no processo de planejamento, execução e avaliação de seu plano de governo e aos cidadãos para que estes se sintam convidados à maior participação na vida pública.

\section{CONCLUSÃO}

O artigo apresentou o desenvolvimento de um software de apuração de custos municipais para o Ensino Fundamental e seus benefícios como ferramenta de apoio à tomada de decisão. O software foi desenvolvido após os trabalhos de $[7,5$, $21,8,16]$ demonstrarem a importância do controle de custos e que uma gestão de custos eficiente melhora o desempenho dos alunos da Educação fundamental. O sistema em seu primeiro módulo, denominado Sistema Integrado de Custos Municipais voltados à Educação (SICM-Educação), colige os fatores que mais contribuem para o custo total do Ensino Fundamental, a saber, o custo de pessoal e merenda. Além de possibilitar o levantamento e cálculo dos custos de cada unidade escolar dos municípios paranaenses, o sistema considera também o desempenho dessas unidades, permitindo assim análises do tipo custo-benefício dos recursos investidos.

A partir de resultados preliminares descritos no artigo com o estudo de caso do município de Assaí, foi possível observar que o sistema possibilita integrar e validar dados que atualmente encontram-se espalhados em diversos setores da prefeitura, como dados provenientes da secretaria municipal de educação, do recursos humanos, da controladoria, entre outros. Tal ação tem potencial de permitir aos gestores públicos uma visão global unificada voltada à tomada de decisão.

A contribuição do SICM-Educação não se restringe ao planejamento e a execução dos planos de governo. O sistema pode ainda subsidiar o controle social, melhorando a transparência à população. Assim, os cidadãos poderão pedir esclarecimentos aos seus governantes, caso os dados apresentados lhes pareçam inadequados. Os dados especí- 
ficos por escola podem permitir desde constatações triviais como o conhecimento dos custos por escola, da quantidade de professores que estão em sala de aula ou em trabalho administrativo, até análises mais elaboradas para o meio acadêmico, como análises de correlação entre as variáveis levantadas pelo sistema.

Apesar de no momento o sistema ter como limitação a apuração de custos das escolas nos anos iniciais do ensino fundamental, a ferramenta poderia ser expandida para as demais séries, aumentando abrangência da ferramenta. Para que isso seja possível alguns problemas deverão ser sanados visto que diferentemente das séries iniciais os outros anos do ensino fundamental não são de responsabilidade dos municípios e sim do estado.

Pode-se então concluir que através da ferramenta desenvolvida, os gestores públicos e a sociedade poderão ter uma visão mais ampla e fidedigna do uso dos recursos usados na Educação Pública, podendo criar ações de melhorias a partir de constatações observadas informações geradas pelo sistema.

\section{REFERÊNCIAS}

[1] F. L. Abrucio. Trajetória recente da gestão pública brasileira: um balanço crítico ea renovação da agenda de reformas. Revista de Administração Pública, 41(spe):67-86, 2007.

[2] M. Alonso. Custo no serviço público. Texto para Discussão, (31), 2008.

[3] E. M. ALVES FILHO and A. L. MARTINEZ. Gestão de custos numa secretaria municipal de educação. In CONGRESSO BRASILEIRO DE CUSTOS, XIII, 2006.

[4] D. Arnott and G. Pervan. Eight key issues for the decision support systems discipline. Decision Support Systems, 44(3):657-672, 2008.

[5] B. Borinelli, S. F. Amâncio-Vieira, L. F. Negreiros, R. S. Pereira, and R. R. G. Pereira. Relação entre custos, desempenho e variáveis educacionais do ensino fundamental: um estudo de caso em ibiporã-pr. Revista do Serviço Público, 65(3):335-354, 2014.

[6] L. C. Bresser Pereira and P. Spink. Gestão do setor público: estratégia e estrutura para um novo estado. 2006.

[7] S. Carvalho, B. Borinelli, V. Suguihiro, S. Vieira, and L. Negreiros. Proposição de metodologia de apuração de custos de unidades escolares para fins de produção de indicadores de gestão de escolas do ensino fundamental (de $1^{\circ}$ ao $4^{\circ}$ ano): um estudo a partir da experiência da cidade de londrina-pr. Tce/Pr.(Org.). Indicadores de Gestão Pública Municipal, pages $15-128,2012$.

[8] D. W. Catelan, R. Martins, D. M. R. de Gouvea, L. F. de Negreiros, S. C. de Carvalho, and S. F. A. Vieira. Custos, desempenho e estrutura educacional do ensino fundamental: uma analise no município de rolândia/pr. 2013.

[9] F. F. das Indústrias do Estado do Rio de Janeiro. Ifdm. [Online; accessed 09-Fevereiro-2015].

[10] A. G. de Almeida and L. Alberton. Gestão de custos na administração pública: aplicação em secretarias municipais de saúde.

[11] A. G. de Almeida, J. A. Borba, and L. C. da Silva Flores. A utilização das informações de custos na gestão da saúde pública: um estudo preliminar em secretarias municipais de saúde do estado de santa catarina. Revista de Administração Pública, 43(3), 2009.

[12] I. I. B. de Geografia e Estatística. Cidades, 2014. [Online; accessed 09-Fevereiro-2015].

[13] C. E. S. DE SALUD. Gestão em hospital de alta complexidade inserido no sistema brasileiro de saúde (sus): Estratégia e custos na área de oncologia. CEP, 1502:001.

[14] W. V. dos Santos. Sistema de informação de custos do governo federal: Modelo conceitual, solução tecnológica e gestão do sistema. In IV Congresso de Gestão Pública, Congresso Nacional de Secretários de Estado de Administração (CONSAD), pages 1-25, 2011.

[15] G. S. G. Leone. Custos - Planejamento, implantação e Controle. Atlas Editora, 3 edition, 2000.

[16] C. d. L. P. Mahnic, J. E. Oliveira, K. T. Semprebom, L. F. Negreiros, B. Borinelli, and S. F. Amâncio-Vieira. Governança e o controle dos custos diretos educacionais nas unidades escolares de ensino fundamental. XX Congresso Brasileiro de Custos., 2013.

[17] A. L. Martinez and E. M. Alves Filho. Sistema de gestão de custos para uma secretaria municipal de saúde. Revista de Contabilidade da UFBA, 6(3):52-65, 2012.

[18] G. S. d. MELLO. Sistema de custos na administração pública-estudo da implantação do método abc em prefeituras de pequeno porte, 2005.

[19] B. R. P. Monteiro, M. C. E. Pereira, W. V. dos Santos, and V. B. de Holanda. O processo de implantação do Sistema de Informação de Custos do Governo Federal do Brasil. In I Congresso Informação de Custo e Qualidade do Gasto no Setor Público, pages 1-14, Brasília, DF, 2010.

[20] R. D. O. MORAES and M. S. S. Calijuri. A implantação de um sistema de custos em uma instituição estadual de ensino superior no estado do paraná. 2006.

[21] L. F. Negreiros, R. S. Pereira, and S. F. A. VIEIRA. Estrutura educacional de ensino fundamental: um estudo comparativo entre dois municípios paranaenses de pequeno e médio porte. ADMpg Gestão Estratégica, 6(2):107-115, 2013.

[22] F. C. Ribeiro, T. P. Ferreira, M. M. de Paula, M. Chaves, S. A. Rodrigues, J. M. de Souza, E. M. Franzosi, and L. F. Oliveira. Vispublica: uma proposta para aprimorar a transparência de dados públicos. Simpósio Brasileiro de Sistemas de Informação, 8:2012, 2012.

[23] L. Secchi. Modelos organizacionais e reformas da administração pública. Revista de Administração Pública, Rio de Janeiro, 43(2):347-369, 2009.

[24] J. C. Vaz, M. M. Ribeiro, and R. Matheus. Dados governamentais abertos e seus impactos sobre os conceitos e práticas de transparência no brasil. Cadernos PPG-AU/UFBA, 9(1), 2011.

[25] M. S. Zanella Di Pietro. Direito administrativo. $2^{\gamma^{a}}$ edição. São, 2000. 